\title{
Anderson Localization Quenches Thermal Transport in Aperiodic Superlattices
}

\author{
Taneli Juntunen," Osmo Vänskä, and Ilkka Tittonen \\ Department of Electronics and Nanoengineering, Aalto University, P.O. Box 13500, FI-00076 Aalto, Finland
}

(Received 25 October 2018; published 12 March 2019)

\begin{abstract}
We show that aperiodic superlattices exhibit intriguing interplay between phononic coherent wave interference effects and incoherent transport. In particular, broadband Anderson localization results in a drastic thermal conductivity reduction of $98 \%$ at room temperature, providing an ultralow value of $1.3 \mathrm{~W} \mathrm{~m}^{-1} \mathrm{~K}^{-1}$, and further yields an anomalously large thermal anisotropy ratio of $\sim 10^{2}$ in aperiodic $\mathrm{Si} / \mathrm{Ge}$ superlattices. A maximum in the thermal conductivity emerges as an unambiguous consequence of phonon Anderson localization at a system length scale bridging the extended and localized transport regimes. The frequency-resolved picture, combined with our lattice dynamical description of Anderson localization, elucidates the rich transport characteristics in these systems and the potential of correlated disorder for sub- to few- $\mathrm{THz}$ phononic engineering of heat transport in thermoelectric applications.
\end{abstract}

DOI: 10.1103/PhysRevLett.122.105901

The recent advent of novel nanophononic systems has enabled tuning the thermal properties of semiconductor materials by the manipulation of coherent thermal phonons $[1,2]$. The currently existing strategies for coherent thermal design rely on two distinct approaches. Phononic metamaterials inhibit thermal transport through local structural resonances, induced via branches [3,4], shells [5], and defect concentrations [6]. Phononic crystals, on the other hand, utilize Bragg resonances from an artificially introduced secondary structural periodicity in the form of interstitial and hole arrays [7] or a superlattice (SL) $[8,9]$. In these systems, effects stemming from the wave nature of phonons enable subwavelength structural features to affect long wavelength phonons with frequencies below 2 THz. Such low-frequency vibrations contribute significantly to thermal transport in standard semiconductor materials due to their long mean-free paths (MFPs) [10]. Consequently, nanophononic engineering opens promising avenues for several technological applications, such as thermoelectrics, where quenching thermal transport without sacrificing the electrical properties of the crystalline material is desirable [11].

Within the class of nanophononic structures, binary SLs are merited by their structural simplicity from an experimental standpoint. Furthermore, SLs provide a natural platform for studying wave effects and the interplay between coherent and incoherent thermal transport at period lengths bridged by the coherence length of the dominant phonon modes [8,9]. Interestingly, theoretical

Published by the American Physical Society under the terms of the Creative Commons Attribution 4.0 International license. Further distribution of this work must maintain attribution to the author(s) and the published article's title, journal citation, and DOI. studies have predicted clear thermal conductivity reduction in SLs with randomized layer thicknesses, including Lennard-Jones [12-14], two-dimensional [15], and SiGebased material systems [16,17]. The phenomenon has been attributed to Anderson localization [18], originating from the destructive interference of coherent phonons and consequently quenching wave transport under structural disorder. Indeed, recent experiments [19] and numerical studies [20] on nanoparticle embedded SLs have shown evidence of Anderson localization of thermal phonons at low temperatures. However, to this end, no studies have been carried out to explicitly elucidate the spectral features of phonon localization in aperiodic SLs under anharmonic interactions. In contrast, aperiodicity has also been suggested as a structural perturbation for destroying lowfrequency phonon coherence [8], offering an alternative interpretation of the earlier works.

In this Letter, we present the spectral characteristics of phonon localization in aperiodic SLs at room temperature, including the effect of incoherent impurity scattering by interfacial mixing of atomic species. We uncover that broadband Anderson localization leads to strongly quenched thermal transport in these systems, resulting in the lowest cross-plane thermal conductivity of $1.3 \mathrm{~W} \mathrm{~m}^{-1} \mathrm{~K}^{-1}$ for $\mathrm{Si} / \mathrm{Ge} \mathrm{SLs}$, well below the alloy limit for $\mathrm{Si}_{0.5} \mathrm{Ge}_{0.5}$. The drastic reduction in cross-plane thermal transport further leads to an anomalously large thermal anisotropy of $\sim 10^{2}$. Moreover, we point out the emergence of a maximum in the thermal conductivity scaling, providing an unambiguous signature of phonon localization for experiments. Finally, we develop a phenomenological expression for phonon transmission covering all relevant transport regimes and provide a complementary lattice dynamical picture elucidating the fundamental origin of Anderson localization in aperiodic SLs. 
As a paradigmatic material system, $\mathrm{Si} / \mathrm{Ge} \mathrm{SLs}$ have been devoted an extensive body of prior theoretical work [2133], including several studies based on molecular dynamics [16,34-38]. As thermal transport in Si/Ge SLs shifts from coherent to incoherent at SL period length of $\sim 4-8 \mathrm{~nm}$ [35], observation of coherent effects necessitates the study of short-period SL systems. Following this rationale, we set up a model system with a period of 10 consecutive atomic layers of $\mathrm{Si}$ and $\mathrm{Ge}$, resulting in a period thickness of $d_{\mathrm{p}}=$ $2.8 \mathrm{~nm}$ for the periodic superlattice (p-SL). To consistently generate aperiodic superlattices (ap-SLs), the ensemble average of which corresponds structurally to $\mathrm{p}-\mathrm{SL}$, we randomly sample ap-SL layer thicknesses within $D$ atomic layers from the nominal thickness of 10 layers, where $D$ then represents a measure of disorder in the periodicity of ap-SL. Figures 1(a) and 1(b) show representative p-SL and ap-SL configurations, respectively, for a system size $L=N_{\mathrm{p}} d_{\mathrm{p}}$, where $N_{\mathrm{p}}$ denotes the number of periods. As surface-segregation driven intermixing of atomic species plays a strong role in thermal transport of experimentally realized SLs [33,35], its effect on localization is further assessed by introducing an atomic mixing region of two atomic layers at the interfaces of SL. The LAMMPS package [39] is utilized for atomistic simulations at a temperature of $300 \mathrm{~K}$ (see Ref. [40] for simulation details).

The overall heat transport in the systems is intuitively described in terms of thermal conductivity $\kappa$. In Fig. 1(c), we present the scaling of cross-plane thermal conductivities for p-SL and ap-SL in the strong disorder limit $(D=9)$ with mixed and sharp interfaces, calculated under nonequilibrium molecular dynamics. The bulk limit $(L \rightarrow \infty)$ is

(a)
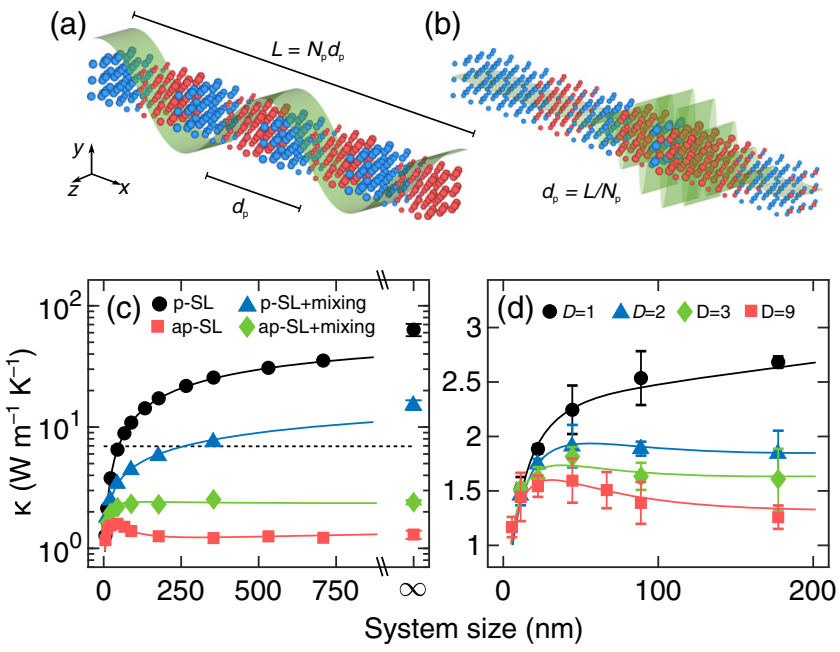

FIG. 1. Schematic images of Si/Ge (a) p-SL and (b) ap-SL, and the associated atom displacement fields for extended and localized phonon modes, respectively. (c) Room-temperature thermal conductivities of $\mathrm{p}-\mathrm{SL}$ and ap-SL in the absence and presence of interfacial mixing as a function of system size. Dashed line represents the bulk alloy of $\mathrm{Si}_{0.5} \mathrm{Ge}_{0.5}$. (d) Emergence of thermal conductivity maximum as disorder increases. Solid lines represent analytic reproductions from the spectral analysis. determined from equilibrium simulations. p-SL exhibits typical scaling behavior in the transition between ballistic $\left(\kappa \propto L^{1}\right)$ and diffusive $\left(\kappa \propto L^{0}\right)$ transport regimes as the system size exceeds the MFPs of the dominant phonon modes, smoothly extrapolating to the bulk value. In p-SL with mixed interfaces, the thermal conductivity shows the same scaling characteristics as with sharp interfaces, however with a considerably reduced value suggesting dominant impurity scattering induced by the atomic mixing.

Strikingly, as we impose aperiodicity on the system with sharp interfaces, thermal conductivity is quenched by a drastic $98 \%$ and shows a nonmonotonic trend at ap-SL size of $\sim 30 \mathrm{~nm}$ as a maximum emerges in the thermal conductivity scaling. In fact, in Fig. 1(d) we show that while the thermal conductivity systematically decreases with increasing aperiodicity, the maximum is observed even in the presence of moderate disorder at $D=2-3$. Indeed, such emergence of a thermal conductivity maximum was recently discovered experimentally [19] and through a Green's function based elastic model [20] at cryogenic temperatures in GaAs/AlAs superlattices with interfacially incorporated ErAs nanoparticles. Here, however, we demonstrate the effect in purely binary layered systems. As we shall discuss below, the maximum originates from the underlying Anderson localization of extended coherent phonon modes as illustrated in Figs. 1(a) and 1(b) (shaded green). Thermal conductivity in the bulk limit is then contributed by nonlocalized diffusive modes. As in-plane thermal conductivity of ap-SL remains comparable to that of p-SL, cross-plane localization additionally provides an anomalously large thermal anisotropy of $\sim 10^{2}$ [40].

Inversely, introducing interfacial mixing into ap-SL smears the maximum thermal conductivity through apparent impurity-induced delocalization. It has been argued that coherence is destroyed by interfacial mixing in $\mathrm{Si} / \mathrm{Ge} \mathrm{SLs}$, which results in the disappearance of the minimum in the period length dependency of thermal conductivity [35], generally regarded as the signature of coherent transport. However, we point out that transport in SLs with interfacial mixing remains coherent for low- to midfrequency phonons, leading to an observable aperiodicity-induced thermal conductivity reduction, well below the alloy limit [dashed line in Fig. 1(c)]. Wave effects should thus be considered when interpreting results from nanophononic systems even in the presence of impurities and dislocations, e.g., as obtained from phonon Monte Carlo simulations which do not conventionally account for coherent phenomena [50].

In previous studies, cross-plane thermal conductivity reduction in random Lennard-Jones multilayer systems has been quantitatively described in terms of a simple twophonon model [12]. In this model, thermal conductivity is composed of an effective exponentially localized coherent mode, as well as an effective incoherent mode insensitive to wave localization. While simple to implement, such decomposition neglects the frequency dependence of MFPs 
and the length scale for localization, and any subexponential incipient frequency regimes between localized and extended transport, often taking the form of a power law [20].

To address the spectral origin of these transport features, we extract the frequency-resolved phonon transmission $\mathcal{T}(\omega)$ in our systems by tracking the force-velocity correlations between atoms during the nonequilibrium simulation [40]. Figures 2(a)-2(d) present phonon transmission spectra for systems of size $L=10-355 \mathrm{~nm}$ for p-SL with sharp and mixed interfaces, and similarly for ap-SL as ensemble averaged over five systems, respectively. The shaded areas in the figures illustrate the different transport regimes as discussed in the following. In both p-SL systems, the transmission at low frequencies $(f<2 \mathrm{THz})$ is length independent and thus contributed by ballistic modes with long anharmonic MFPs. For the length-dependent transmission of the diffusive modes above $2 \mathrm{THz}$, interfacial disorder promotes strongly increased scattering, resulting in the thermal conductivity reduction in Fig. 1(c).

The observed remarkable performance of ap-SL with sharp interfaces, however, results from the broadband

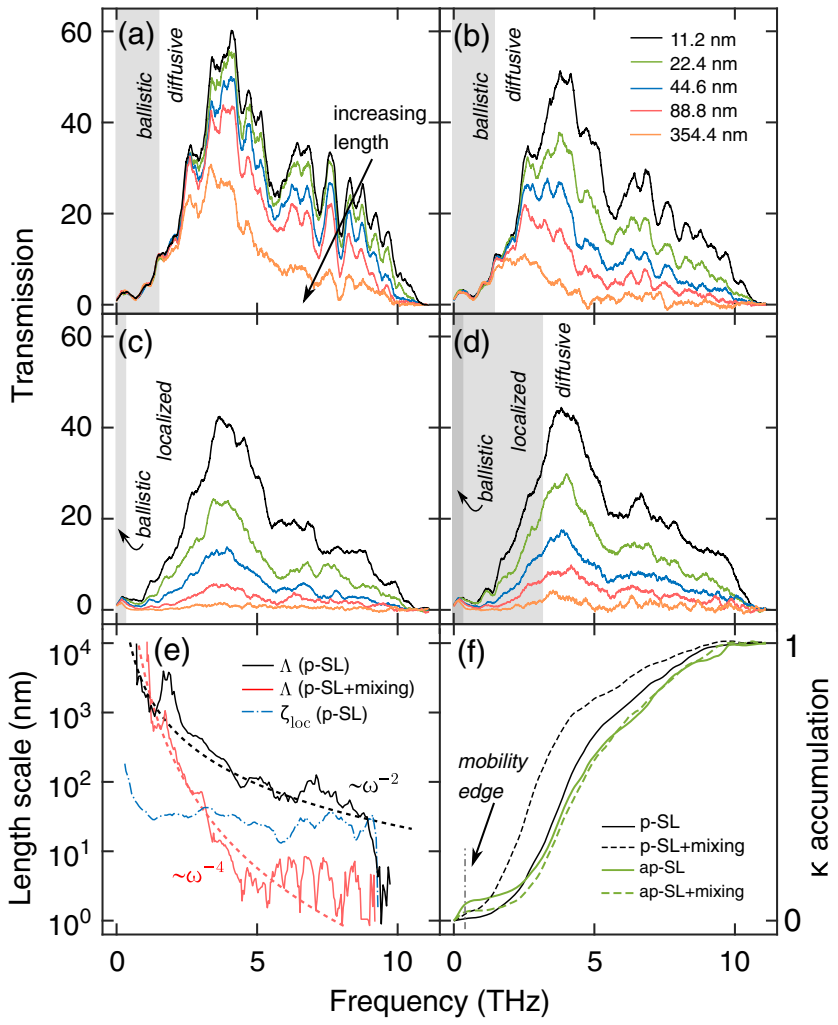

FIG. 2. Frequency-resolved phonon transmission at different system sizes of (a),(b) p-SL and (c),(d) ap-SL with sharp and mixed interfaces, respectively. (e) Phonon MFPs and localization lengths at $300 \mathrm{~K}$. Dashed lines represent fits to power law $\sim \omega^{\alpha}$, with $\alpha=-2$ and -4 indicating phonon-phonon and impurity scattering, respectively. (f) Normalized thermal conductivity accumulation $(L=354.4 \mathrm{~nm})$. extinction of phonon transmission [Fig. 2(c)] as compared to $\mathrm{p}$-SL, down to $\sim 0.4 \mathrm{THz}$ thus identified here as the mobility edge. The phonon transmission reduction shows the characteristic exponential size dependency of Anderson localization throughout the phonon spectrum in the 0.4-9 $\mathrm{THz}$ frequency range [40]. The finding suggests a ballistic-to-localized transition for the low-frequency coherent spectral contribution. Such transition was experimentally observed only recently [19], now pointed out through our atomistic simulations. As a testimony to the wave origin of the phenomenon, Anderson localization affects transport only up to $\sim 4 \mathrm{THz}$ [Fig. 2(d)] within interfacially mixed ap-SL, after which the available modes follow the diffusive behavior of interfacially mixed p-SL. Thus, incoherent scattering induces dephasing, delocalizing the high-frequency modes. As impurity scattering provides a weaker thermal conductivity reduction than Anderson localization, the thermal conductivity of ap-SL in Fig. 1(c) increases with the inclusion of interfacial mixing.

In general terms, the long wavelength phonons below $2 \mathrm{THz}$ present a fundamental challenge for thermal design as they cannot be scattered by nanoscale features, such as guest-material interstitials or surface roughness. Our spectral findings suggest that aperiodicity-driven phonon localization presents a highly robust strategy for tuning the midto low-frequency phonons without fabricating structurally complex features, such as resonant branches or pillars $[3,4]$. The low thermal conductivity is further achieved without deteriorating the crystalline structure of the material, which is optimal for retaining good electrical properties in, e.g., thermoelectric applications [51].

We illuminate the interplay between different transport regimes through the characteristic length scales in the systems, presented in Fig. 2(e). The anharmonic MFPs $\Lambda$ of phonons follow from the length-dependent transmission functions of $\mathrm{p}$-SL (denoted by superscript $\mathrm{p}$ ) $\mathcal{T}^{\mathrm{p}}(\omega)=\left\{\left[\mathcal{T}_{0}(\omega)\right] /(1+L /[2 \Lambda(\omega)])\right\}$, where we treat $\mathcal{T}_{0}$ and $\Lambda$ as fitting parameters [52,53]. In case of sharp interfaces, MFPs scale as $\sim \omega^{-2}$, indicating that diffusive transport is dominated by phonon-phonon scattering. Here the interfaces act as a source for Bragg scattering, allowing for interactions associated with wave dynamics. Introducing atomic mixing into p-SL yields $\sim \omega^{-4}$ scaling as a signature of Rayleigh scattering by atomic impurities [54], resulting in the dramatic reduction of the high-frequency MFPs. Meanwhile, the low-frequency ballistic regime in Figs. 2(a) and 2(b) is characterized by MFPs of several micrometers. Given the exponential behavior associated with Anderson localization, we extract the frequency-resolved cross-plane localization lengths $\xi_{\text {loc }}$ from $\overline{\mathcal{T}}^{\mathrm{ap}}(\omega) / \mathcal{T}^{\mathrm{p}}(\omega)=e^{-L / \xi_{\text {loc }}(\omega)}$, where superscript ap refers to ap-SL and the overline denotes an ensemble-averaged quantity. The localization length diverges at the mobility edge, decreasing down to $\sim 30 \mathrm{~nm}$ where the maximum thermal conductivity in Fig. 1(c) is observed. $\xi_{\text {loc }}$ then coincides with $\Lambda$ of 
interfacially mixed p-SL at $4 \mathrm{THz}$, marking the frequency after which modes are delocalized by impurity scattering in agreement with Fig. 2(d). As a result, while the localization length is found roughly independent of the interface quality below $4 \mathrm{THz}$, no exponential character may be assigned to higher frequencies for systems with interfacial mixing.

The normalized thermal conductivity accumulation in Fig. 2(f) reveals the spectral origin of heat transport in apSL; a full analysis of the thermal conductivity accumulation is presented in Ref. [40]. While localization generates a plateau above the mobility edge at $f>0.4 \mathrm{THz}$, we find that high-frequency modes, undergoing localization, retain finite heat transport even in large systems with a length scale of several $\xi_{\text {loc }}$. Such transition from fully to partially localized states coincides with the reduction in the coherence length of SL as extracted from the spatial crosscorrelation function of atomic velocities [40]. This incipient localization regime suggests that aperiodicity promotes sensitivity to phase breaking processes for high-frequency phonons with a spatial coherence length comparable to the period of SL.

To combine our gathered insight on the heat transport across the frequency spectrum in the form of an analytic expression, we propose a phenomenological model accounting for the interplay between phonon localization and phase breaking processes in ap-SL. In the model, we modify the argument of the exponential,

$$
\ln \left(\frac{\overline{\mathcal{T}}^{\mathrm{ap}}(\omega)}{\mathcal{T}^{\mathrm{p}}(\omega)}\right)=-\frac{L}{\xi_{\mathrm{loc}}(\omega)} \frac{l_{\phi}(\omega)}{L+l_{\phi}(\omega)}
$$

where $l_{\phi}$ denotes the frequency-dependent characteristic length scale for the dephasing of the phonons in the incipient localization regime. The expression is reminiscent of the phenomenological approach utilized for the ballisticto-diffusive transition of $\mathcal{T}^{\mathrm{p}}(\omega)$ [52,53], now smoothly interpolating between the localized and extended transport regimes through a power-law-like behavior. Here, we briefly describe the implications of Eq. (1) for the relative thermal conductivity $\kappa^{\prime} \equiv \bar{\kappa}^{\mathrm{ap}} / \kappa^{\mathrm{p}} \propto \overline{\mathcal{T}}^{\text {ap }} / \mathcal{T}^{\mathrm{p}}$. It is straightforward to see that in the incoherent limit, $l_{\phi} \ll \xi_{\text {loc }}$, the exponential reverts to unity $\left(\kappa^{\prime}=1\right)$ and transport is unaffected by localization. On the other hand, given the localization criterion, $l_{\phi} \gg \xi_{\text {loc }}$, the standard exponential form of $\kappa^{\prime} \propto e^{-L / \xi_{\text {loc }}}$ is assumed and transport is eventually quenched as the system size increases. However, in the incipient regime when $l_{\phi} \sim \xi_{\text {loc }}$, the exponential converges to a finite value between zero and unity, $\kappa^{\prime} \propto e^{-l_{\phi} / \xi_{\text {loc }}}$. Thus, $l_{\phi}$ replaces $L$ in the exponential expression, providing a cutoff for system size beyond which localization no longer occurs. As the presented expression covers the behavior of the complete frequency spectrum, we provide analytic reproductions of the simulation results based on the
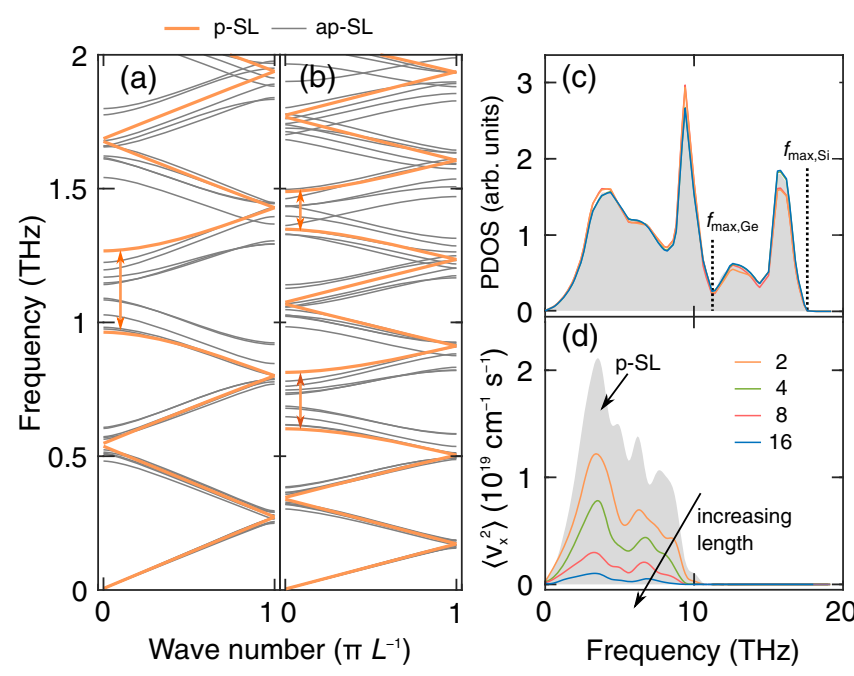

FIG. 3. Low-frequency cross-plane phonon dispersion of p-SL (thick line) and five ap-SL system configurations (thin lines) for (a) longitudinal and (b) transverse phonons with $N_{\mathrm{p}}=4$. (c) Phonon density of states. The maximum frequencies of the vibrational spectra of $\mathrm{Ge}$ and $\mathrm{Si}$ are indicated by dashed lines. (d) Group velocity weighted phonon density of states for p-SL and ap-SL with $N_{\mathrm{p}}=2-16$.

extracted MFPs in conjuction with Eq. (1) as solid lines in Figs. 1(c) and 1(d). The agreement is benign.

Finally, we set the localization phenomenon on a more fundamental basis via lattice dynamical calculations [40]. Figures 3(a) and 3(b) present the low-frequency phonon dispersion in the cross-plane direction in p-SL with $N_{\mathrm{p}}=4$, as well as in five representative ap-SLs for longitudinal and transverse phonons, respectively. In the absence of aperiodicity, the phonon dispersion shows the characteristic phononic gaps (indicated by arrows) of coherent SLs, insensitive to the size of the system. The cross-plane group velocity reduction near the gaps marks the well-established origin of the thermal conductivity minimum in SLs [55]. In aperiodic systems, however, increasing the system size not only folds the Brillouin zone onto itself, but drives further miniband formation and group velocity reduction at the newly formed Brillouin zone edges. As a result, while the ensemble-averaged phonon density of states (PDOS) shown in Fig. 3(c) remains unaffected by aperiodicity, the average group velocity assigned to the states is drastically reduced in ap-SL. To elucidate this effect, in Fig. 3(d) we weight the PDOS by the square of the respective cross-plane group velocities $v_{\mathbf{k}, \nu}^{x}$ to obtain $\left\langle v_{x}^{2}\right\rangle=V^{-1} \sum_{\mathbf{k}, \nu} \delta\left(\omega-\omega_{\mathbf{k}, \nu}\right)\left|v_{\mathbf{k}, \nu}^{x}\right|^{2}$, where $V$ is the volume and $\mathbf{k}$ and $\nu$ denote the wave vector and phonon branch, respectively. While modes above $11 \mathrm{THz}$ are trapped into the Si layers as they are not supported by the Ge constituent, an additional broadband quenching of the group velocity is driven by secondary gap formation. The finding is in agreement with the strong decrease in the atomic participation of the thermal phonon modes within 
ap-SL, while the in-plane group velocities are found insensitive to aperiodicity [40]. Interestingly, early theoretical work on SLs interpreted the thermal conductivity minimum to originate from localizationlike behavior [56]. Even though the effect was later shown to result from the competition between gap formation and incoherent processes, the present work reassociates phonon Anderson localization with secondary phononic miniband formation.

In light of the lattice dynamical picture and as discussed in detail in Ref. [40], the exponential behavior associated with Anderson localization is thus contained in the system size dependence of the phonon group velocities. Since increasing decoherence closes the phononic gaps in the SL phonon dispersion [55], the dephasing length $l_{\phi}$ provides an upper cutoff for system size after which the miniband formation no longer occurs. Indeed, such an interpretation is in agreement with Eq. (1).

In conclusion, Anderson localization is a powerful tool for broadband quenching of phonon transport, particularly in the sub- to few- $\mathrm{THz}$ range, leading to an ultralow crossplane thermal conductivity at room temperature, thus beating the alloy limit without affecting in-plane transport or the crystalline integrity of the system. We accurately reproduce the simulated thermal conductivities using a phenomenological model covering the characteristics of the full transport spectrum, as well as show that localization is driven by secondary miniband formation. We expect the results to translate to a wide range of periodic nanostructures exhibiting the thermal conductivity minimum, such as 2D [15], nanowire [41], and twinning [38] superlattices, offering new insight for the flexible optimization of thermal properties. Finally, the emergence of thermal conductivity maximum is expected to be a key experimental feature for understanding wave localization in novel phononic systems, readily within reach by the use of the currently existing fabrication techniques.

Simulations were performed using computational resources provided by CSC-IT Center for Science, Finland, and Aalto Science-IT project. T. J. acknowledges ELEC Doctoral School for financial support. This work was performed, in part, within TransFlexTeg under European Union's Horizon 2020 programme (No. 645241) and Academy of Finland Project No. 285972.

*taneli.juntunen@aalto.fi

[1] M. Maldovan, Nature (London) 503, 209 (2013).

[2] S. Volz et al., Eur. Phys. J. B 89, 15 (2016).

[3] B. L. Davis and M. I. Hussein, Phys. Rev. Lett. 112, 055505 (2014).

[4] S. Xiong, K. Sääskilahti, Y. A. Kosevich, H. Han, D. Donadio, and S. Volz, Phys. Rev. Lett. 117, 025503 (2016).

[5] J. Chen, G. Zhang, and B. Li, J. Chem. Phys. 135, 104508 (2011).
[6] T. Zhu, K. Swaminathan-Gopalan, K. J. Cruse, K. Stephani, and E. Ertekin, Adv. Funct. Mater. 28, 1706268 (2018).

[7] J.-K. Yu, S. Mitrovic, D. Tham, J. Varghese, and J. R. Heath, Nat. Nanotechnol. 5, 718 (2010).

[8] M. N. Luckyanova, J. Garg, K. Esfarjani, A. Jandl, M. T. Bulsara, A. J. Schmidt, A. J. Minnich, S. Chen, M. S. Dresselhaus, Z. Ren, E. A. Fitzgerald, and G. Chen, Science 338, 936 (2012).

[9] J. Ravichandran, A. K. Yadav, R. Cheaito, P. B. Rossen, A. Soukiassian, S. J. Suresha, J. C. Duda, B. M. Foley, C.-H. Lee, Y. Zhu, A. W. Lichtenberger, J. E. Moore, D. A. Muller, D. G. Schlom, P. E. Hopkins, A. Majumdar, R. Ramesh, and M. A. Zurbuchen, Nat. Mater. 13, 168 (2014).

[10] A. S. Henry and G. Chen, J. Comput. Theor. Nanosci. 5, 141 (2008).

[11] T. Juntunen, H. Jussila, M. Ruoho, S. Liu, G. Hu, T. Albrow-Owen, L. W. Ng, R. C. Howe, T. Hasan, Z. Sun, and I. Tittonen, Adv. Funct. Mater. 28, 1800480 (2018).

[12] Y. Wang, H. Huang, and X. Ruan, Phys. Rev. B 90, 165406 (2014).

[13] Y. Wang, C. Gu, and X. Ruan, Appl. Phys. Lett. 106, 073104 (2015).

[14] P. Chakraborty, L. Cao, and Y. Wang, Sci. Rep. 7, 8134 (2017).

[15] T. Zhu and E. Ertekin, Phys. Rev. B 90, 195209 (2014).

[16] B. Qiu, G. Chen, and Z. Tian, Nanosc. Microsc. Therm. 19, 272 (2015).

[17] R. Frieling, S. Eon, D. Wolf, and H. Bracht, Phys. Status Solidi A 213, 549 (2016).

[18] P. W. Anderson, Phys. Rev. 109, 1492 (1958).

[19] M. N. Luckyanova, J. Mendoza, H. Lu, B. Song, S. Huang, J. Zhou, M. Li, Y. Dong, H. Zhou, J. Garlow, L. Wu, B. J. Kirby, A. J. Grutter, A. A. Puretzky, Y. Zhu, M. S. Dresselhaus, A. Gossard, and G. Chen, Sci. Adv. 4, eaat9460 (2018).

[20] J. Mendoza and G. Chen, Nano Lett. 16, 7616 (2016).

[21] P. Hyldgaard and G. D. Mahan, Phys. Rev. B 56, 10754 (1997).

[22] G. Chen, Phys. Rev. B 57, 14958 (1998).

[23] S.-i. Tamura, Y. Tanaka, and H. J. Maris, Phys. Rev. B 60, 2627 (1999).

[24] A. A. Kiselev, K. W. Kim, and M. A. Stroscio, Phys. Rev. B 62, 6896 (2000).

[25] H. Zhao and J. B. Freund, J. Appl. Phys. 97, 024903 (2005).

[26] S.-F. Ren, W. Cheng, and G. Chen, J. Appl. Phys. 100, 103505 (2006).

[27] A. Ward and D. A. Broido, Phys. Rev. B 77, 245328 (2008).

[28] S. P. Hepplestone and G. P. Srivastava, Phys. Rev. B 84, 115326 (2011).

[29] J. Garg, N. Bonini, and N. Marzari, Nano Lett. 11, 5135 (2011).

[30] I. O. Thomas and G. P. Srivastava, Phys. Rev. B 88, 115207 (2013).

[31] I. Savić, D. Donadio, F. Gygi, and G. Galli, Appl. Phys. Lett. 102, 073113 (2013).

[32] J. Garg and G. Chen, Phys. Rev. B 87, 140302 (2013).

[33] P. Chen, N. A. Katcho, J. P. Feser, W. Li, M. Glaser, O. G. Schmidt, D. G. Cahill, N. Mingo, and A. Rastelli, Phys. Rev. Lett. 111, 115901 (2013).

[34] S. Volz, J. B. Saulnier, G. Chen, and P. Beauchamp, Microelectron. J. 31, 815 (2000). 
[35] E. S. Landry and A. J. H. McGaughey, Phys. Rev. B 79, 075316 (2009).

[36] V. Samvedi and V. Tomar, J. Appl. Phys. 105, 013541 (2009).

[37] Y. Chalopin, K. Esfarjani, A. Henry, S. Volz, and G. Chen, Phys. Rev. B 85, 195302 (2012).

[38] H. Dong, B. Wen, Y. Zhang, and R. Melnik, RSC Adv. 7, 29959 (2017).

[39] S. Plimpton, J. Comput. Phys. 117, 1 (1995).

[40] See Supplemental Material at http://link.aps.org/ supplemental/10.1103/PhysRevLett.122.105901, which includes Refs. [35,39,41-49], for simulation details, equilibrium results and thermal anisotropy, characteristic length scales and thermal conductivity accumulation, and modal analysis of thermal phonons.

[41] M. Hu and D. Poulikakos, Nano Lett. 12, 5487 (2012).

[42] F. H. Stillinger and T. A. Weber, Phys. Rev. B 31, 5262 (1985).

[43] K. Ding and H. C. Andersen, Phys. Rev. B 34, 6987 (1986).

[44] R. Kubo, M. Toda, and N. Hashitsume, Statistical Physics II: Nonequilibrium Statistical Mechanics (Springer-Verlag, Berlin, 2012), Vol. 31.
[45] J. Chen, G. Zhang, and B. Li, Phys. Lett. A 374, 2392 (2010).

[46] C. H. Baker and P. M. Norris, Phys. Rev. B 91, 180302 (2015).

[47] K. Sääskilahti, J. Oksanen, J. Tulkki, and S. Volz, Phys. Rev. B 90, 134312 (2014).

[48] K. Sääskilahti, J. Oksanen, S. Volz, and J. Tulkki, Phys. Rev. B 91, 115426 (2015).

[49] B. Latour, S. Volz, and Y. Chalopin, Phys. Rev. B 90, 014307 (2014).

[50] Q. Li and W. Ye, Int. J. Heat Mass Transfer 107, 534 (2017).

[51] M. Ruoho, T. Juntunen, and I. Tittonen, Nanotechnology 27, 355403 (2016).

[52] S. Datta, Electronic Transport in Mesoscopic Systems (Cambridge University Press, Cambridge, England, 1997).

[53] J. Wang and J.-S. Wang, Appl. Phys. Lett. 88, 111909 (2006).

[54] P. G. Klemens, Solid State Physics (Academic Press, New York, 1958), Vol. 7.

[55] M. V. Simkin and G. D. Mahan, Phys. Rev. Lett. 84, 927 (2000).

[56] R. Venkatasubramanian, Phys. Rev. B 61, 3091 (2000). 